\title{
Submitted from BMJ Quality \\ Reducing the occurrence of errors in a laboratory's specimen receiving and processing department
}

\author{
Nouf Al Saleem, Khaled AI-Surimi
}

To cite: Al Saleem N, Al-Surimi K. Reducing the occurrence of errors in a laboratory's specimen receiving and processing department. BMJ Quality Improvement Reports 2016;5:u211474.w4624 doi:10.1136/bmjquality. u211474.w4624

Received 27 April 2016 Revised 10 June 2016

\section{CrossMark}

King Saud bin Abdulaziz University for Health Sciences /King Abdullah International Medical Research Center, Riyadh, Saudi Arabia

Correspondence to Nouf Al Saleem alsaleem. nouf@gmail.com

\section{ABSTRACT}

Frequent, preventable medical errors can have an adverse effect on patient safety and quality as well as leading to wasted resources. In the laboratory, errors can occur at any stage of sample processing; preanalytical, analytical, and post analytical stages. However evidence shows most of the laboratory errors occur during the pre-analytical stage. The receipt and processing of specimens is one of the main steps in the pre-analytical stage. Errors in this stage could be due to mislabeling, incorrect test entry and entering the wrong location, among other reasons. Most of these errors are preventable. At the Riyadh Regional Laboratory of the Ministry of Health, we found that there was an average of 2.31 errors per 1000 processed samples; these errors had occurred during the pre-analytical stage. These samples were returned back from other laboratory departments, such as Chemistry, Hematology and Microbiology, to the receiving and processing department. We decided to carry out an improvement project where we applied a systematic approach to identify and analyse the root causes of the problem using quality tools such as a process flowchart and a fish-bone diagram. The Model for Improvement was used and several PDSA (Plan, Do, Study, Act) cycles were run to test interventions which aimed to prevent laboratory processing errors and mistakes. The project results showed a $25 \%$ reduction in errors during the pre-analytical stage.

\section{PROBLEM}

The specimen receiving and processing department is the key entry point at the Riyadh Regional Laboratory (RRL) of the Ministry of Health. This regional laboratory is one of the largest reference laboratories for the Ministry of Health. RRL consists of around 14 departments. The specimen receiving and processing department is the largest department with around 35 employees. ${ }^{1}$ The RRL receives around 2000 samples a day from all of the Kingdom regions. All samples are processed by the specimen receiving and processing department before they are sent to the corresponding laboratory sections for the required tests. Errors in specimen processing can lead to adverse events which include; delays in patient diagnosis and management. Based on a retrospective analysis of data, we noticed that at RRL there were a number of dispatched specimens which were returned back to the receiving and processing department on a daily basis. We analysed a random sample of historical data and found that there were 2.31 errors per 1000 dispatched samples. These samples were sent back due to processing errors and mistakes which included; mislabelling, wrong test entry, wrong location, unjustified delay, and also processing STAT specimens as routine samples. Thus, we decided to undertake a quality improvement project which aimed to reduce the occurrence of defects in the specimen processing department by $50 \%$ over a three months period. This was a step towards aiming for zero error.

\section{BACKGROUND}

Since 2000, medical errors have been given significant attention around the world to assure the safety of healthcare. In the book 'To Err Is Human', it is stated that medical errors are responsible for around 58\% of adverse events and that sadly, most of these events are preventable. ${ }^{2}$ The American Center for Disease Control and Prevention (CDC) has documented that laboratory results affect around $70 \%$ of the decision making for patients' management. ${ }^{3}$ A number of previous studies showed that most laboratory errors occur in the pre-analytical phase of patient investigations, ${ }^{4}$ and one of the most common errors is when the patient is not identified correctly. Laboratory error is also an issue of patient safety; the Joint Commission have the identification of patients as their first international patient safety goal (IPSG).$^{5}$

A study by Cavenaugh found that laboratory error can result in high costs to organisations. Thus, reducing laboratory errors can increase the efficiency of the laboratory. ${ }^{6}$ 


\section{BASELINE MEASUREMENT}

We collected data retrospectively for 12 working days from the department's logbook. The rate of error in the sample was calculated to be 2.31 per 1000 . We divided the number of errors (60 errors) by the number of total samples received $(60 / 25,942)$. Missing data from the logbook were excluded. Figure 1 shows the data analysis and the number of processing errors.

\section{DESIGN}

The project team included the project leader, the head of department, the laboratory supervisor and two of the senior technicians in the department. The team was led by a quality management specialist.

The team undertook a root cause analysis using the fish-bone diagram and drawing out the department's work flow. The fish-bone diagram showed different areas of error related to people, methods, processes, and culture. The main reasons for error were related to staff training, adherence to policy, work schedule, supervision, and absence of disciplinary action.

Based on these processing errors, several solutions were proposed for testing using PDSA cycles (Plan, Do, Study, Act). The proposed intervention included the following steps: providing a list of tests for each laboratory section, assigning a senior staff member to each unskilled staff member for each entering section in the receiving department and sharing the number of errors with the employees to support them in learning from their mistakes.

The main outcome measure was the number of returned samples from the laboratory sections to the receiving and processing department. The balancing measures were staff satisfaction around sharing and learning from mistakes and with their new work flow.

\section{STRATEGY}

PDSA (1) aimed to improve accuracy of entering the sample process correctly by providing a list of the tests' names and codes for all entering sections in the department. We also assigned a second staff member to

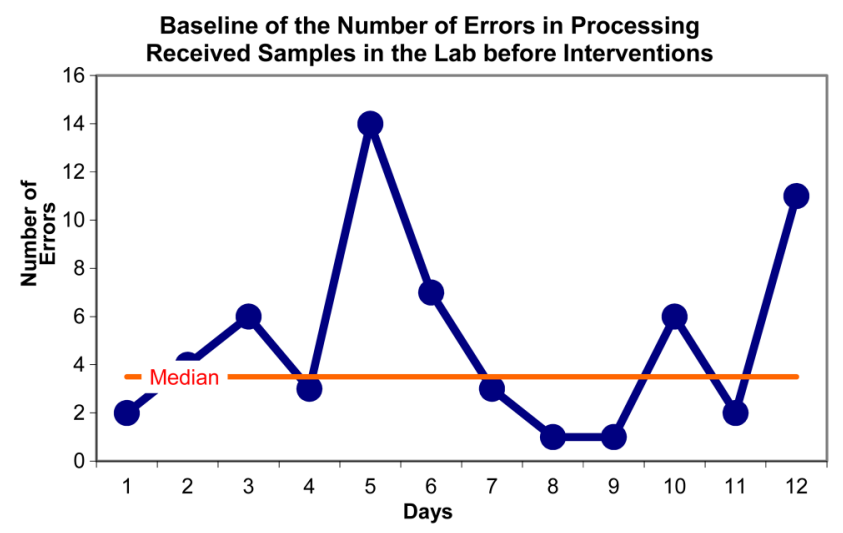

Figure 1 perform a quality control check before sending specimens to the lab sections. Our prediction was that the proposed intervention would help staff to be aware of the codes of tests to prevent incorrect entry and mislabeled samples. The study stage analysis showed that entering errors were still increasing. Although staff were provided with the list and were informed of the importance of checking before sending for processing, staff were not complying, which did not meet our prediction. These results led us to conclude that informing and training staff was not enough, other ideas needed to be tested.

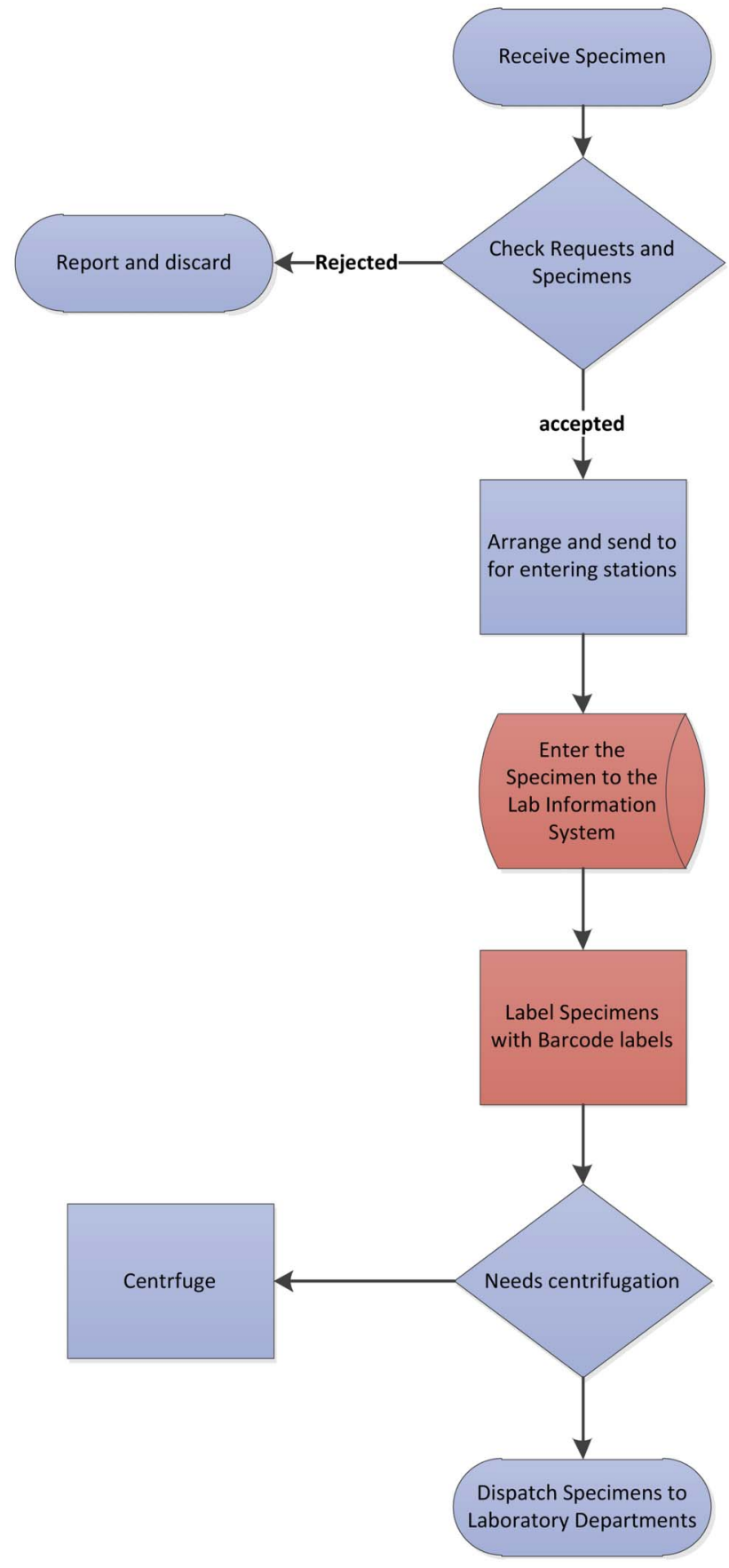

Figure 2 Flow chart of specimen processing 
Figure 3 Project Data

\begin{tabular}{|c|c|c|c|c|c|c|c|}
\hline \multicolumn{4}{|c|}{ Baseline measurement } & \multicolumn{4}{|c|}{ Post intervention measurement } \\
\hline $\begin{array}{l}\text { No. } \\
\text { Days }\end{array}$ & $\underline{\text { Date }}$ & $\frac{\begin{array}{c}\text { No. of } \\
\text { Mistakes }\end{array}}{\text { /day }}$ & $\frac{\begin{array}{c}\text { Total } \\
\text { Specimen }\end{array}}{/ \text { day }}$ & $\begin{array}{l}\text { No. } \\
\text { Days }\end{array}$ & Date & $\frac{\begin{array}{c}\text { No. of } \\
\text { Mistakes }\end{array}}{\text { /day }}$ & $\begin{array}{c}\text { Total No. } \\
\text { Specimen/day }\end{array}$ \\
\hline 1 & $13 / 10$ & 2 & 8,173 & 13 & $\begin{array}{l}\text { PDSA \#1 } \\
11 / 11\end{array}$ & 6 & 2,176 \\
\hline 2 & $14 / 10$ & 4 & 1,915 & 14 & $12 / 11$ & 2 & 2,256 \\
\hline 3 & $15 / 10$ & 6 & 2,097 & 15 & $13 / 11$ & 6 & 1,989 \\
\hline 4 & $16 / 10$ & 3 & 2,076 & 16 & $\begin{array}{l}\text { PDSA \#2 } \\
14 / 11\end{array}$ & 5 & 1,896 \\
\hline 5 & $17 / 10$ & 14 & 1,799 & 17 & $17 / 11$ & 4 & 2,017 \\
\hline 6 & $20 / 10$ & 7 & 2,008 & 18 & $18 / 11$ & 3 & 2,091 \\
\hline 7 & $21 / 10$ & 3 & 1,901 & 19 & $19 / 11$ & 3 & 2,023 \\
\hline 8 & $30 / 10$ & 1 & 1,803 & 20 & $20 / 11$ & 2 & 1,902 \\
\hline 9 & $31 / 10$ & 1 & 1,901 & 21 & $\begin{array}{l}\text { PDSA \#3 } \\
21 / 11\end{array}$ & 1 & 2,247 \\
\hline 10 & $6 / 11$ & 6 & 2,023 & 22 & $24 / 11$ & 3 & 2,157 \\
\hline 11 & $7 / 11$ & 2 & 1,973 & 23 & $25 / 11$ & 0 & 1,876 \\
\hline 12 & $10 / 11$ & 11 & 1,897 & 24 & $26 / 11$ & 3 & 2,203 \\
\hline \multicolumn{2}{|c|}{$\begin{array}{l}\text { Total no of days: } \\
12 \text { days }\end{array}$} & 60 & 25,942 & \multicolumn{2}{|c|}{$\begin{array}{l}\text { Total no of days: } \\
12 \text { days }\end{array}$} & 38 & 24,833 \\
\hline \multicolumn{2}{|c|}{$\begin{array}{l}\text { Average mistakes } \\
\text { per day }\end{array}$} & \multicolumn{2}{|c|}{5} & \multicolumn{2}{|c|}{$\begin{array}{l}\text { Average of } \\
\text { mistakes }\end{array}$} & \multicolumn{2}{|r|}{3.0} \\
\hline \multicolumn{2}{|c|}{$\begin{array}{l}\text { Percentage of } \\
\text { mistakes out total } \\
\text { specimen s }\end{array}$} & \multicolumn{2}{|c|}{$0.2 \%$} & \multicolumn{2}{|c|}{$\begin{array}{l}\text { Percentage of } \\
\text { mistakes }\end{array}$} & \multicolumn{2}{|r|}{$0.15 \%$} \\
\hline \multicolumn{2}{|c|}{ The difference } & & kes $\mathrm{p}$ & repre & enting 25 & uction & rror concurrence \\
\hline
\end{tabular}

Figure 4

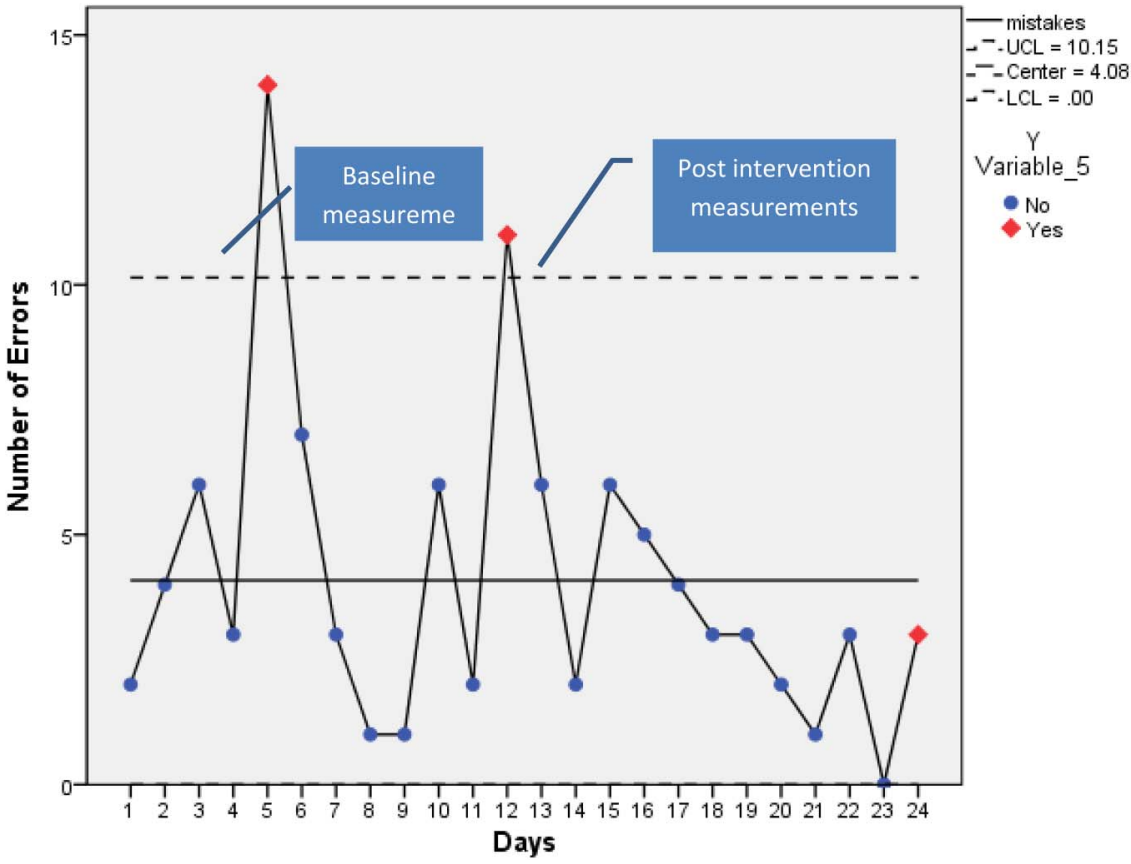

PDSA (2): Based on the results of PDSA (1), we decided to test the idea of sharing with staff, the number and type of errors they had made. We started by testing the idea on two staff members, informing them about the number and type of errors they had made over the last month. We expected that after having been told about the errors that staff would be monitored and further action taken if appropriate. The study stage showed that staff were willing to improve. Our results showed a decrease in error occurrence, which supported the idea of having a monitoring and evaluation mechanism in place for work performance. As a result, we adapted the intervention of reporting, sharing, and learning from mistakes and decided in the next PDSA to roll this out for all staff.

PDSA (3) tested the idea of sharing errors with all staff members by informing them about the number and type of mistakes they had made in the last month and after the project started. We expected that all staff would show a willingness to learn from their mistakes. 
The staff immediately reacted positively, showing their willingness to improve and to learn from their mistakes. Later, the results showed a noticeable decrease in the number of errors and mistakes revealing the importance of having a monitoring and evaluation system in place as well as the benefits of sharing and learning from mistakes.

\section{RESULTS}

The project results, as presented in the control chart, showed a trend of reduction in the number of errors which continued six points after the intervention. The overall impact of the project was to reduce the frequency of errors by $25 \%$ in the first 12 days of the post implementation period in comparison to the baseline measurement period. On average during the baseline period there were 5 errors $(0.2 \%)$ compared with only 3 errors $(0.15 \%)$ in the post intervention period. This improvement showed a steady trend decrease in error occurrence until reaching zero error in day 23 of the post intervention period. However, on day 24 there was another error occurrence which was still below the average and within the control process limits, suggesting that there was a common cause of variation which needed to be analysed and corrected. We believe that the results of this project will help to ensure patient safety and also avoid wasting valuable resources in RRL at two levels: direct costs from labour and materials (through avoiding extra work for employees), and indirect costs from misdiagnosis and mismanagement of patients.

\section{LESSONS AND LIMITATIONS}

We have learnt an important lesson through this project and that is that giving training, whilst necessary, it is not sufficient to improve staff performance. It is vital to hold people accountable for their performance, and to put effective monitoring and evaluation systems in place. Sharing and learning from mistakes can help to improve culture as teams become aware of the consequences of errors on patient care and safety.

The project has been undertaken over a short period of time, however the tested interventions appear to have given promising results. The results are generalisable and could be replicated in other similar departments. To try and ensure sustainability, we presented and discussed the project results with the higher management team of RRL. The management team showed interest in the project's results and showed a willingness to adapt the interventions as part of routine daily practice in the department. These simple change ideas could be replicated and tested further in other laboratories in the country.

\section{CONCLUSION}

We involved staff in the project by showing them their mistakes and allowing them to be part of the solution. This allowed us to achieve our improvement aim. The project results emphasise the importance of workflow monitoring and evaluation to increase staff compliance with quality assurance measures. As well as improving the quality of laboratory services, we hope that patient safety and efficiency will also improve.

Acknowledgements I would like to thank the Head of Specimens and Processing Department in Riyadh Regional Laboratory, Mr. Saad Al Suraya, for his great support to improve the department's performance and for his valuable suggestions of the possible solutions.

Thanks go to Dr. Ahmed Al Amry, the Head of Quality Management Department in King Abdullah Medical City, National Guard for his valuable information and knowledge he shared regarding Quality Improvement Model and his successful experience in his organization.

Finally, a special thanks for Dr. Khaled Al-Surimi for his support and guidance during the whole project.

Declaration of interests None declared.

Ethical approval The problem was chosen based on the importance and the frequency of the mistakes. This problem is related to the core mission and duties of the specimen receiving and processing department, and fits with the Riyadh Regional Laboratory vision and mission to provide a high quality of patient services. This meets the $\mathrm{JCI}$ international standards and CBAHI national standard as well. Thus the head of the department approved the project and participated in it as well. All information relating to the staff who were involved in performing the process were kept private and confidential.

Open Access This is an open-access article distributed under the terms of the Creative Commons Attribution Non-commercial License, which permits use, distribution, and reproduction in any medium, provided the original work is properly cited, the use is non commercial and is otherwise in compliance with the license. See:

- http://creativecommons.org/licenses/by-nc/2.0/

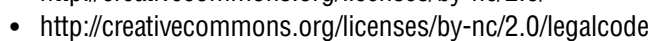

\section{REFERENCES}

1. Al Saleem N, El Metwally A, Househ M. Electronic Lab Information Exchange (ELIE) in Saudi Arabia. Stud Health Technol Inform. 2014; 202:134-7.

2. Kohn L.T., Corrigan J.M., and Donaldson M.S. To err is human: building a Safer Health System. Vol. 6. 2000: National Academies Press.

3. Moiduddin P.D.a.A., White Paper: Electronic Exchange of Clinical Laboratory: Information Issues and Opportunities. 2008.

4. Bonini P., et al., Errors in laboratory medicine. Clinical chemistry, 2002. 48: p. 691-698.

5. JCAOH and Joint Commission Resources, Meeting the Joint Commission's 2007 National Patient Safety Goals. 2006: Joint Commission Resources.

6. Cavenaugh, Edward Lee. "A method for determining costs associated with laboratory error." American journal of public health $1981 ; 71.8: 831-4$ 